\title{
PREVALENCIA DE Salmonella y ENTEROPARÁSITOS EN ALIMENTOS Y MANIPULADORES DE ALIMENTOS DE VENTAS AMBULANTES Y RESTAURANTES EN UN SECTOR DEL NORTE DE BOGOTÁ, COLOMBIA
}

\section{PREVALENCE OF Salmonella AND ENTEROPARASITES IN FOOD AND FOOD STALLS HANDLERS AND RESTAURANTS IN AN AREA OF THE NORTH OF BOGOTA, COLOMBIA}

\author{
Martín A. Bayona R. ${ }^{1}$ \\ ${ }^{1}$ Bacteriólogo, Esp., M.Sc. Facultad de Medicina, Universidad de Ciencias Aplicadas y Ambientales U.D.C.A. Calle 222 No. \\ 55-37, Bogotá, D.C. E-mail: mabayona@udca.edu.co
}

Rev. U.D.C.A Act. \& Div. Cient. 15(2): 267 - 274, 2012

\section{RESUMEN}

Las enfermedades transmitidas por alimentos representan una problemática mundial. La vigilancia de los víveres comercializados es un tema que debe llevar a tomar conciencia del riesgo sanitario y de la importancia en la implementación de acciones correctivas, en la capacitación y en el seguimiento de los manipuladores de alimentos. El presente trabajo correspondió a un estudio descriptivo de prevalencia, en donde se evaluó la frecuencia de ciertos microorganismos en los manipuladores y alimentos vendidos, en una zona del norte de Bogotá, referente a la carga de enteroparásitos y Salmonella spp. La población evaluada correspondió a 60 manipuladores de alimentos distribuidos así: 40, de venta establecida y 20 , de venta ambulatoria, quienes aceptaron el consentimiento informado. Se realizaron ensayos microbiológicos en muestras de heces, de frotis de manos y de alimentos en la búsqueda de enteroparásitos y Salmonella spp. Se realizó una estadística descriptiva. Para procesar los datos, se empleó el programa SPSS versión 18. Dentro de los resultados, se destaca que el $25 \%$ de los alimentos ambulantes y el $7,5 \%$ de los alimentos de venta establecida (chorizo frito, ensaladas de frutas, yogurth con cereal, arepa rellena y pincho de carne) fueron positivos para Salmonella spp. La presencia de diferentes parásitos, como Entamoeba histolytica / Entamoeba dispar, Giardia intestinalis, Cryptosporidium spp. y Ascaris lumbricoides representan un aspecto de importancia en salud pública, lo que implica socializar los resultados obtenidos y fomentar una cultura de higiene en los manipuladores de alimentos.
Palabras clave: Alimentos, buenas prácticas de manipulación, Salmonella, parásitos, salud pública.

\section{SUMMARY}

Foodborne diseases are a global problem. Surveillance of marketed food is an issue that should carry health risk awareness and the importance in the implementation of corrective actions, training and monitoring of food handlers. The present research corresponded to a descriptive study that assessed prevalence in the frequency of certain microorganisms in food handlers and sold in an area of northern Bogota, regarding the burden of enteric and Salmonella spp. The population was composed of 60 food handlers distributed as follows: 40 of established sites and 20 of ambulatory sales; all agreed to the informed consent. Microbiological tests were performed on fecal samples, food and hand smear in the search for enteroparasites and enteric Salmonella spp. Descriptive statistics were performed. To process the data SPSS version 18 was used. Among the results it can be highlighted that $25 \%$ of the food sold in the street and $7.5 \%$ of the permanent food expenditures (fried sausage, fruit salads, yogurt with cereal, stuffed corn pancake and shish kebab) were positive for Salmonella spp. The presence of parasites such as Entamoeba histolytica / Entamoeba dispar, Giardia intestinalis, Cryptosporidium spp. and Ascaris lumbricoides represent an aspect of importance for public health, implying the need to socialize the results and foster a culture of hygiene in food handlers. 
Key words: Food, Good manipulation practices, Salmonella, parasites, public health.

\section{INTRODUCCIÓN}

Para muchos peatones, la venta de alimentos generada por vendedores ambulantes satisface la necesidad básica de comida, mientras se encuentran fuera del hogar o del sitio de trabajo, sin darse cuenta que los productos pueden ser una fuente de infección, debido a su inadecuada manipulación. Existe una relación directa entre la inadecuada manipulación de los alimentos y la asociación de Enfermedades Transmitidas por alimentos (ETA), en la cual, el manipulador interviene como vehículo de transmisión (Gubbay et al. 2004).

Al observar la alta frecuencia de brotes de intoxicación por alimentos son múltiples los factores que contribuyen a este efecto; entre ellos, se destacan el inadecuado aseo de los manipuladores, la obtención de materias primas contaminadas, la refrigeración incorrecta de los alimentos terminados y de las materias primas, una deficiente limpieza y desinfección de los equipos y utensilios de trabajo y la localización de los sitios de trabajo, asociados a contaminación ambiental (Merino, 2008; Vásquez et al. 2007).

La carencia de estudios de epidemiología alimentaria en Colombia, que muestran la importancia de los manipuladores asociados con ETA, impide tener datos actualizados sobre la distribución de los microorganismos asociados con patologías gastrointestinales; además, que su desconocimiento no permite establecer medidas de vigilancia epidemiológica y toma de decisiones en salud pública (Flórez et al. 2008).

La prevalencia de ciertos microorganismos patógenos en los manipuladores es enorme, tal como lo describe Valdivieso et al. (2006). La limitada oferta de trabajo en las grandes ciudades, junto a la falta de capacitación y las necesidades sentidas de la población, lleva a algunas personas a buscar alternativas, como vendedores ambulantes, para la obtención de ingresos económicos (Durango et al. 2004).

En Colombia, en el 2010, se notificaron al sistema nacional de vigilancia por archivos planos (colectivo) 11.563 casos de ETA, involucrados en 761 brotes. Los alimentos más frecuentes asociados con brotes de ETA fueron: alimentos mixtos seguidos por queso, arroz con pollo, carne y sus derivados y productos de pesca y sus derivados. El lugar de consumo implicado en la ocurrencia de brotes de ETA correspondió al hogar (49\%), seguido de establecimiento educativo (14\%) y restaurante comercial $(9 \%)$. Los agentes etiológicos más prevalentes detectados en muestras biológicas, en alimentos, en superficies y en manipuladores de alimentos fueron: Staphylococcus aureus, Escherichia coli,
Salmonella spp. y hongos entre otros (Instituto Nacional de Salud, 2011a y b).

De acuerdo con Vásquez et al. (2007), la frecuencia de las ETA se proyecta al incremento, debido a la urbanización, modos de vida y modificación de los hábitos alimentarios de la población. La tendencia generalizada a tomar los alimentos fuera del hogar, por la jornada de trabajo y por el estudio, obligan al consumo de alimentos en diferentes establecimientos. Muchos alimentos comercializados, se pueden contaminar, adquiriendo así características altamente peligrosas, por constituir un transporte potencial para los patógenos. Estos alimentos, generalmente, requieren una estricta refrigeración y, debido a su composición o características físicas, químicas o biológicas favorecen el crecimiento de microorganismos y la formación de sus toxinas, desarrollando gran afinidad al deterioro, como son los embutidos, productos de la pesca, lácteos, carnes y huevos (Durango et al. 2004; Rincón et al. 2011). Los alimentos son vectores de más de 200 enfermedades incrementándose en países, como el nuestro, donde factores socioeconómicos, culturales y sanitarios, se incluyen en programas de vigilancia y de seguridad alimentaria (Vargas et al. 2004; Díaz et al. 2006). Las enfermedades parasitarias producidas por protozoos y helmintos representan un problema importante de salud pública. Estas enfermedades afectan a más de dos billones de personas de la población mundial (Vázquez \& Campos, 2009; Sánchez, 2006).

Las especies de Salmonella spp. son los miembros más complejos de la familia Enterobacteriaceae, con aproximadamente 2.400 serotipos descritos hasta la fecha. Actualmente, el género se clasifica en dos especies: S. enterica y $S$. bongori (Vásquez et al. 2007). Este género es patógeno para humanos y para animales, se adquieren por vía oral, causando enteritis, infección sistémica y fiebre entérica y se asocian con la ingesta de alimentos mal preparados o manipulados inadecuadamente (Rodríguez \& Prado, 2006; Rodulfo et al. 2012; Rincón et al. 2011).

La salmonelosis es una zoonosis ampliamente distribuida a nivel mundial. Las infecciones por Salmonella spp. pueden causar pequeños brotes en la población; entre 60 y $80 \%$ de los casos son esporádicos, aunque, a veces, se producen grandes brotes en hospitales, jardines infantiles, hogares geriátricos y restaurantes (Yánez et al. 2008). Salmonella enterica ocasiona, en la mayoría de los casos, una enfermedad autolimitada, pero en personas jóvenes, en mayores o en inmunosuprimidas puede alcanzar la gravedad y causar la muerte (Irvine et al. 2009); este microorganismo esta asociado, frecuentemente, con enfermedad diarreica aguda, propagándose, principalmente, por la ingesta de alimentos, de aguas contaminadas o por personas infectadas asociadas a la manipulación de alimentos (Uribe \& Suárez, 2006; Rodulfo et al. 2012). 
Teniendo en cuenta el gran número de establecimientos que expenden alimentos en un sector del norte de Bogotá surgió el interés por conocer la frecuencia de microrganismos, como Salmonella spp. y enteroparásitos en manipuladores $\mathrm{y}$ alimentos vendidos en este sector.

\section{MATERIALES Y MÉTODOS}

El presente trabajo correspondió a un estudio descriptivo, no probabilístico, a conveniencia del investigador, quien evaluó la prevalencia de enteroparásitos y Salmonella spp., en alimentos y en manipuladores de alimentos. La población evaluada estuvo conformada por 60 manipuladores de alimentos de ambos géneros, distribuidos de la siguiente manera: 40 , procedentes de restaurantes establecidos y 20 , de venta ambulante, cercanos a un Centro Universitario, comprendidos entre la calle 170 a la calle 222 (sector norte de Bogotá). La población fue escogida por conveniencia, con base en un consentimiento informado. Se excluyeron aquellos manipuladores que hubiesen recibido tratamiento con antibacterianos y antiparasitarios el último mes.

Obtención de las muestras: De cada uno de los manipuladores, se recolectaron tres muestras: una, del alimento que estaba preparando al momento de la evaluación; un frotis de manos, incluidas las uñas y dos muestras de materia fecal, en donde se analizaron: coprológico (enteroparásitos) y coprocultivo (Salmonella spp.).

Alimentos: Se tomaron por duplicado $200 \mathrm{~g}$ y se envasaron en recipientes plásticos estériles, para luego ser transportados al laboratorio de Microbiología.

Manos: La muestra, se tomó por contacto directo en agar MacConkey (Mc) y, posteriormente, se realizó un hisopado con agua peptonada $0,1 \%$ estéril para después ser analizada en la búsqueda de enteroparásitos.

Heces: Previa explicación, dada a los manipuladores sobre la toma de muestra, se recolectaron heces de los manipuladores de alimentos en dos días diferentes, para su posterior transporte al laboratorio, en un lapso no mayor de 2 horas.

Análisis Parasitológico: Las muestras, se analizaron macroscópicamente, mediante observación simple del aspecto, consistencia, color, presencia de moco y sangre y parásitos adultos. Para el examen microscópico, se empleó la técnica de sedimentación espontánea en tubo (TSET) y, posteriormente, se realizaron montajes en lámina de vidrio, empleando lugol parasitológico y coloración de Ziehl Neelsen modificada (Rodríguez \& Prado, 2006).

Bacteriológico: Se realizaron cultivos directos de materia fecal (coprocultivo) en los medios: Salmonella - Shigella (SSA), Xilosa Lisina Desoxicolato (XLD) y Agar Mac Conkey (Mc). Las placas, se incubaron a $37^{\circ} \mathrm{C}$, durante 24 - 48 horas, con una humedad del $90 \%$; de igual manera, se llevó a cabo un enriquecimiento de las muestras de materia fecal en caldo selenito, el cual, se incubó a $37^{\circ} \mathrm{C}$, durante $6-18$ horas, a partir del cual, se practicaron subcultivos en los medios anteriormente mencionados. Posteriormente, se aplicó una evaluación macroscópica de las colonias y se determinó la identificación de género y de especie, mediante un micro método cualitativo, que emplea sustratos convencionales y cromogénicos, para la identificación de Enterobacterias (Roberts et al. 2000; Vargas et al. 2004).

Para el análisis del frotis de manos, se sembraron las muestras directamente y por estriación sobre los medios de cultivo XLD y MC, siguiendo los pasos anteriores. De las muestras de alimentos, se pesaron $25 \mathrm{~g}$ y se homogenizaron con $225 \mathrm{~mL}$. de agua peptonada (1\%); luego, se incubaron a $37^{\circ} \mathrm{C}$, por 24 horas; a partir de allí, se introdujo $1 \mathrm{~mL}$ de cada muestra en $9 \mathrm{~mL}$ de caldo Rappaport y caldo tetrationato; posteriormente, se efectuó un subcultivo en medios de SSA, Hecktoen y XLD; estas muestras fueron incubadas, a $37^{\circ} \mathrm{C}$, por 24 horas. Las colonias sospechosas de pertenecer a Salmonella spp. fueron diagnosticadas, mediante pruebas bioquímicas miniaturizadas (Vargas et al. 2004).

Aspectos éticos y legales: Durante la realización del presente trabajo, se obtuvo el consentimiento informado de cada uno de los manipuladores de alimentos; de igual manera, no se reveló la identidad de los manipuladores de alimentos, ni de las entidades en donde se recolectaron las muestras.

Se realizó una estadística descriptiva y para el procesamiento de los datos, se empleó el programa estadístico para las ciencias sociales (SPSS) versión 18.

\section{RESULTADOS Y DISCUSIÓN}

De los manipuladores de alimentos de restaurantes establecidos, se destacan los siguientes resultados (Tabla 1). A partir de los coprológicos, se presentaron los siguientes hallazgos: Blastocystis hominis, $30 \%$ en manipuladores ambulantes y establecidos; quistes de Giardia. Intestinalis, 5\% y 2,5\%, respectivamente, en manipuladores ambulantes y establecidos; huevos de Ascaris lumbricoides, $10 \%$ y 2,5\%, respectivamente; complejo Entamoeba histolytica / Entamoeba dispar, $25 \%$ y $10 \%$, respectivamente, en manipuladores ambulantes y establecidos. De acuerdo con lo anterior, se evidencia que los manipuladores de alimentos presentan parásitos intestinales representativos, lo cual, es relevante en la manipulación de alimentos.

El trabajo realizado por Rincón et al. (2009) contrasta con el presente estudio, en cuanto a la gran diversidad de enteroparásitos encontrados en manipuladores de alimentos. Este 
Tabla 1. Resultados obtenidos entre los manipuladores de alimentos y los parámetros: coprológico, coprocultivo, frotis de manos y alimentos, en un sector de norte de Bogotá.

\begin{tabular}{|c|c|c|c|c|c|c|c|}
\hline \multirow{3}{*}{\multicolumn{2}{|c|}{ Parámetros evaluados }} & \multicolumn{6}{|c|}{ Sitio de Venta } \\
\hline & & \multicolumn{3}{|c|}{ Ambulante } & \multicolumn{3}{|c|}{ Establecido } \\
\hline & & & $\%$ del $N$ & & & $\% \operatorname{del} N$ & \\
\hline \multirow{8}{*}{ Coprológico } & 0 & 5 & 25,0 & 18,5 & 22 & 55,0 & 81,5 \\
\hline & B. hominis & 6 & 30,0 & 33,3 & 12 & 30,0 & 66,7 \\
\hline & E. histol-E. dispar & 5 & 25,0 & 55,6 & 4 & 10,0 & 44,4 \\
\hline & Salmonella & 0 & ,0\% & , 0 & 0 & ,0 & ,0 \\
\hline & H. Ascaris & 2 & 10,0 & 66,7 & 1 & 2,5 & 33,3 \\
\hline & Q. Giardia & 1 & 5,0 & 50,0 & 1 & 2,5 & 50,0 \\
\hline & C. parvum & 1 & 5,0 & 100,0 & 0 & ,0 & ,0 \\
\hline & Q. E. coli & 0 & ,0 & ,0 & 0 & ,0 & ,0 \\
\hline \multirow{8}{*}{ Coprocultivo } & 0 & 17 & 85,0 & 32,1 & 36 & 90,0 & 67,9 \\
\hline & B. hominis & 0 & ,0 & ,0 & 0 & ,0 & ,0 \\
\hline & E. histol-E. dispar & 0 & ,0 & ,0 & 0 & ,0 & ,0 \\
\hline & Salmonella & 3 & 15,0 & 42,9 & 4 & 10,0 & 57,1 \\
\hline & H. Ascaris & 0 & ,0 & ,0 & 0 & ,0 & ,0 \\
\hline & Q. Giardia & 0 & 0 & 0 & 0 & 0 & 0 \\
\hline & C. Parvum & 0 & 0 & 0 & 0 & 0 & 0 \\
\hline & Q. E. coli & 0 & ,0 & ,0 & 0 & ,0 & 0 \\
\hline \multirow{8}{*}{ Frotis de manos } & 0 & 15 & 75,0 & 28,3 & 38 & 95,0 & 71,7 \\
\hline & B. Hominis & 3 & 15,0 & 60,0 & 2 & 5,0 & 40,0 \\
\hline & E. histol-E. dispar & 1 & 5,0 & 100,0 & 0 & ,0 & ,0 \\
\hline & Salmonella & 1 & 5,0 & 100,0 & 0 & ,0 & , 0 \\
\hline & H. Ascaris & 0 & ,0 & ,0 & 0 & ,0 & ,0 \\
\hline & Q. Giardia & 0 &, 0 & ,0 & 0 & ,0 & ,0 \\
\hline & C. parvum & 0 &, 0 & ,0 & 0 & ,0 & ,0 \\
\hline & Q. E. coli & 0 &, 0 & ,0 & 0 & , 0 & ,0 \\
\hline \multirow{8}{*}{ Alimentos } & 0 & 15 & 75,0 & 28,8 & 37 & 92,5 & 71,2 \\
\hline & B. hominis & 0 & ,0 & , 0 & 0 & ,0 & ,0 \\
\hline & E. histol-E. dispar & 0 & ,0 & ,0 & 0 & ,0 & ,0 \\
\hline & Salmonella & 5 & 25,0 & 62,5 & 3 & 7,5 & 37,5 \\
\hline & H. Ascaris & 0 & ,0 & , 0 & 0 & 0 & , 0 \\
\hline & Q. Giardia & 0 & ,0 & ,0 & 0 & , 0 & ,0 \\
\hline & C. parvum & 0 & ,0 & ,0 & 0 & , 0 & ,0 \\
\hline & Q. E. coli & 0 & ,0 & ,0 & 0 & ,0 & ,0 \\
\hline
\end{tabular}


autor destaca que la especie más frecuente reportada de los helmintos fue A. lumbricoides (1,5\%), seguido por Trichuris trichiura $(0,5 \%)$ y Uncinaria (0,5\%). Dentro de los protozoos patógenos, el parásito que más se detectó fue el complejo de E. histolytica / E. dispar (14,6\%) y G. intestinalis (2,4\%). Diferente a este estudio, se considera el hallazgo del protozoo, cuyo poder patógeno es aún controvertido, como es $B$. hominis $(1,4 \%)$ y de parásitos comensales, como Endolimax nana $(18,14 \%)$, E. coli $(6,52 \%)$ y una asociación de $E$. nana y $E$. coli del $(3,7 \%)$; todas las muestras fueron analizadas para coccidios intestinales (Cryptosporidium spp., Isospora belli, Cyclospora spp), obteniéndose resultados negativos. En el presente trabajo solamente se obtuvo un individuo con Cryptosporidium spp. y se resalta el hecho de encontrar un alto porcentaje (30\%) de B. hominis.

El hecho de encontrar $B$. hominis en alimentos indica que los manipuladores han tenido una deficiente práctica en la manipulación de los alimentos y, por consiguiente, los han contaminado; éste parásito patógeno potencial se asocia en muchos casos con patología intestinal en pacientes inmunocomprometidos, de acuerdo con los trabajos realizados por Port et al. (2004); Rincón et al. (2009) y Pérez et al. (2008).

El presente estudio concuerda, parcialmente, con aquel realizado por Gómez et al. (1999), quienes llevaron a cabo una investigación epidemiológica descriptiva en manipuladores de alimentos, de los principales centros de expendio de alimentos, en la ciudad de Santa Clara (Cuba), identificando G. intestinalis (55,4\%), E. histolytica (25,3\%), Enterobius vermicularis (14,5\%) y T. trichiura y A. lumbricoides (2,4\%). La diferencia del presente estudio con el anterior, se obtuvo en que no identificamos E. vermicularis, T. trichiura y el porcentaje de G. intestinalis solamente correspondió al 5\%.

Las amebas no patógenas, como $E$. coli encontradas en frotis de manos de los manipuladores de alimentos en el presente estudio es un indicativo de malos hábitos higiénicos, lo que concuerda con resultados similares de la investigación adelantada por Port et al. (2004), en Brasil, donde el parásito con mayor prevalencia en manipuladores de alimentos de hospitales públicos y privados fue también $E$. coli seguido de $E$. nana. A pesar que estas especies no son patógenas y no representan un gran impacto clínico son de gran relevancia epidemiológica, ya que muestra que el individuo parasitado puede ser un foco latente de contagio (Cortés et al. 2008).

A partir del coprocultivo en que se buscó la presencia de Salmonella spp., se obtuvo una prevalencia del 15 y $10 \%$ en manipuladores ambulantes y restaurantes, respectivamente. Se destaca que dentro de las salmonelas identificadas una cepa correspondió al serotipo Typhi y tres al Enteritidis, de acuerdo con la identificación bioquímica, por el método miniaturizado empleado.
La salmonelosis es una infección que puede ser asintomática, lo que genera un gran problema porque es altamente contagiosa, debido a la baja dosis infectante. Principalmente, es relevante en manipuladores de alimentos, los cuales, podrían ser portadores y diseminar el microorganismo (Fica et al. 2001). Los manipuladores con un coprocultivo positivo para Salmonella spp. no presentaron manifestaciones gastrointestinales, siendo así un riesgo para los consumidores.

Las infecciones en humanos producidas por Salmonella spp., en general, son causadas por la ingestión de alimentos contaminados por excrementos humanos o de animales infectados (Uribe \& Suárez, 2006).

La ingesta de alimentos y de aguas contaminadas con dicho microrganismo, conlleva a una gastroenteritis pero, así mismo, puede ocasionar enfermedad extra - intestinal (Nataro et al. 2007; Rincón et al. 2011). Los brotes de ETA asociados con Salmonella spp., se producen, generalmente, por la ingestión de carne vacuna, derivados aviares, huevos o productos lácteos; de igual forma, se han asociado con algunos productos, como melones, tomates, ensaladas de verduras crudas, jugo de naranja no pasteurizado (Vásquez et al. 2007).

A partir del frotis de manos, se destaca que el 15\% de los manipuladores de venta establecidos fueron positivos en $15 \%$, para $B$. hominis; $5 \%$, para el complejo E. histolytica / E. dispar y 5\%, Salmonella spp., indicando que los manipuladores de alimentos presentan restos de heces en sus manos, lo cual, podría comprometer la salud de los consumidores. Estos datos concuerdan con el trabajo de Peralta \& Ayala (2008), quienes afirman que las enfermedades producidas por protozoarios intestinales son causa de alta morbilidad, su distribución es alta en todo el territorio nacional, pero se desconoce su prevalencia actual.

Flórez et al. (2008) determinaron los factores relacionados con enfermedades transmitidas por alimentos en restaurantes de cinco ciudades de Colombia, se encontraron importantes deficiencias en el cumplimiento de las buenas prácticas de elaboración de alimentos, en los hábitos de los manipuladores y en las condiciones sanitarias de los expendios de alimentos. Estos hallazgos implican la urgencia o necesidad de capacitación de los manipuladores en estos aspectos y fortalecer, desde la autoridad sanitaria, la inspección adecuada de las condiciones de calidad de los establecimientos y de las prácticas higiénicas (Instituto Nacional de Salud, 2011, a y b).

Después de los alimentos analizados, se evidenció que el $25 \%$ de los alimentos de venta ambulante y el 7,5\% de los alimentos de venta establecida (chorizo frito, ensaladas de frutas, yogurt con cereal, arepa rellena y pincho de carne) fue- 
ron positivos para Salmonella spp., representando un riesgo para la salud del consumidor. Dichos resultados concuerdan con el trabajo reportado por Bayona (2009), en donde se obtuvo el $25 \%$ de Salmonella spp. en alimentos vendidos en la vía pública, confirmando aún más el riesgo que representan ciertos alimentos, como la ensalada de frutas y los chorizos, reportados en el presente trabajo.

Con relación a Salmonella spp. aislada a partir de alimentos, el presente estudio coincide con el trabajo de Cavalcante et al. (2011), quienes al analizar muestras de jamón completamente cocido, encontraron que un $30 \%$ de las muestras estaban contaminadas con este microrganismo; los datos muestran la necesidad de una mejora en el control de calidad en todas las partes de los sistemas alimentarios; de igual manera, el presente estudio coincide con el trabajo reportado por Méndez et al. (2011), quienes al analizar 42 muestras de alimentos provenientes de venta ambulante en un sector de Chapinero en la ciudad de Bogotá, en el 2010, reportaron una prevalencia de Salmonella enterica no tifoidea, de $11,1 \%$, en productos tales como hamburguesas y arepa con chorizo.

De acuerdo con los resultados obtenidos, se confirma la importancia atribuida a los manipuladores de alimentos, sin discriminar los de venta ambulante y de restaurantes, para lo cual, no se encontró ninguna asociación, de acuerdo con la prueba de Chi cuadrado.

En la mayoría de los casos, el personal seleccionado contratado para la manipulación de alimentos no es el más apropiado, puesto que no tienen conocimientos adecuados para realizar estas prácticas y aún con la capacitación del personal, la manipulación de los alimentos, tanto en crudo como en producto terminado no es el más adecuado, como lo confirman en sus trabajos Flórez et al. (2008); Durango et al. (2004); Pedregal et al. (2002); Bayona (2009) y Valdivieso et al. (2006).

Los manipuladores de alimentos de venta ambulante deberán asistir al médico como control; sin embargo, en el caso específico de los manipuladores de ventas callejeras, el $100 \%$ argumenta que no asisten periódicamente, salvo en extrema urgencia, ya que se ven afectados en sus ingresos.

La eliminación inadecuada de las aguas residuales y la basura derivadas de la venta callejera de alimentos incrementan el potencial de transmisión de enfermedades microbianas, al estimular la proliferación de insectos y de roedores transmisores de enfermedades entéricas (Pedregal et al. 2002).

De acuerdo con Quispe \& Sánchez (2001), la correcta higiene de los alimentos esta determinada por diversos factores, entre los que se encuentran las condiciones de obtención de los mismos, características de los medios empleados para su transporte, temperaturas y condiciones de almacenamiento, estructura de los locales, donde se manipulan los alimentos y las prácticas de los manipuladores.

El presente estudio coincide con el trabajo descrito por Vásquez et al. (2007), cuyo objetivo consistió en identificar los problemas sanitarios existentes en los servicios de alimentación de instituciones infantiles del Instituto Colombiano de Bienestar Familiar (ICBF), asociados con manipuladores. Los resultados microbiológicos evidenciaron deficiencias higiénicas sanitarias confirmando, aún más, los actuales resultados. De acuerdo con lo anterior, se resalta la importancia de formalizar un control sanitario estricto y permanente por las autoridades competentes, la implementación de los programas del plan de saneamiento básico y la capacitación en buenas prácticas de manufactura para todos los manipuladores, lo cual, garantiza la inocuidad y la calidad de los alimentos, para proteger la salud y el bienestar de la población infantil.

La presencia de patógenos, como son E. histolytica / E. dispar, G. intestinalis, Cryptosporidium spp. y A. lumbricoides en heces de manipuladores de alimentos representan un riesgo de importancia, a nivel de salud pública, lo cual, nos compromete como comunidad académica a difundir y a socializar estos resultados, con el fin de promocionar y de prevenir las enfermedades, que estos microorganismos producen.

El presente trabajo determinó la prevalencia de microorganismos a los que estamos expuestos los consumidores, ya que una gran cantidad de la población bogotana se ve en la necesidad de consumir alimentos fuera del hogar, acudiendo a restaurantes y a puestos ambulantes de ventas de alimentos, donde se ingieren diferentes productos, sin que se guarden las normas y los procedimientos que se deben tener para la elaboración, la preparación, el consumo y la distribución de alimentos.

Agradecimientos. El autor agradece el apoyo brindado por la Universidad de Ciencias Aplicadas y Ambientales U.D.C.A, a la Facultad de Medicina y a los docentes: Dra. María Teresa Ríos, Dr. Fernando Gallego y Dr. Elkin Higuera, por sus orientaciones y valiosos aportes con el programa estadístico. Financiación: El trabajo fue financiado por la Universidad de Ciencias Aplicadas y Ambientales U.D.C.A. Conflictos de interés: El autor declara que no existe ningún conflicto de interés que ponga en riesgo la validez de los resultados presentados.

\section{BIBLIOGRAFÍA}

1. BAYONA, M. 2009. Evaluación microbiológica de alimentos adquiridos en la vía pública en un sector 
del norte de Bogotá. Rev. U.D.C.A Act. \& Div. Cient. 12(2):9-17.

2. CAVALCANTE, A.; TEIXEIRA, E.; FRIZZO, S.; DE SOUZA, N.; CAVALCANTE, A.; MONTENEGRO, T. 2011. Salmonella sp e Listeria monocytogenes em presunto suíno comercializado em supermercados de Fortaleza (CE, Brasil): fator de risco para a saúde pública. Ciênc. Saúde Coletiva.16(2):657-662.

3. CORTÉS, D.; ESTRADA, M.; ÁREAS K.; SIERRA, A. 2008. Frecuencia de parasitismo intestinal en expendedores de alimentos ubicados en los recintos de la UNAN-León. Universitas. 2:25-28.

4. DÍAZ, T.; CABALLERO, A.; DÍAZ, J.; CARDONA, M.; MOREJÓN, P.; SÁNCHEZ, Y. 2006. Estudio, control y prevención de las ETA: infección e intoxicación por alimentos. Instituto de Nutrición e Higiene de los Alimentos, Cuba. Rev. Soc. Ven. Microbiol. 26:95-100.

5. DURANGO, J.; ARRIETA, G.; MÁTTAR, S. 2004. Presencia deSalmonella spp en un área del Caribe Colombiano: un riesgo para la salud pública. Biomédica. 24:89-96.

6. FICA, C.; ALEXANDRE, M.; PRAT, S.; FERNÁNDEZ, A.; FERNÁNDEZ, J.; HEITMANN, I. 2001. Cambios epidemiológicos de la salmonelosis en Chile. Desde Salmonella typhi a Salmonella enteritidis. Rev. Chil. Infect. 18(2):85-93.

7. FLÓREZ, A.; RINCÓN, C.; GARZÓN, P.; VARGAS, N.; ENRÍQUEZ, C. 2008. Factores relacionados con enfermedades transmitidas por alimentos en restaurantes de cinco ciudades de Colombia, 2007. Infectio (Colombia). 12(4):255-266.

8. GÓMEZ, M.; ORIHUELA, J.; ORIHUELA, M.; FERNÁNDEZ, N. 1999. Parasitismo intestinal en manipuladores de alimentos. Rev Cubana Med. Gen. Integr. 15(5):520-523.

9. GUBBAY, L.; GALANTERNIK, L.; GALAN, G.; CABRERA, J.; DURANGO, M. 2004. Staphylococcus aureus: sensibilidad antibiótica y detección de enterotoxinas de cepas aisladas en alimentos y manos de manipuladores. Rev. Cien. Venezuela. 30:4-12.

10. INSTITUTO NACIONAL DE SALUD. 2011 a. Informe de evento: Enfermedades transmitidas por alimentos, hasta el período epidemiológico 13 del año 2011. Disponible desde Internet en: http:www.ins.gov.co (con acceso 26/09/12).
11. INSTITUTO NACIONAL DE SALUD. 2011 b. Vigilancia y control en Salud pública: Protocolo de vigilancia y control de enfermedades transmitidas por alimentos. Disponible desde Internet en: http:www.ins.gov. co (con acceso 26/09/12).

12. IRVINE, W.; GILLESPIE, I.; SMITH, F.; ROONEY, P.; MCCLENAGHAN, A.; DEVINE, M.; TOHANI, V. 2009. Investigation of outbreak of Salmonella enterica serovar Newport infection. Epidemiol. Infect. 137:1449-1456.

13. MÉNDEZ, I.; BADILLO, C.; ORTÍZ, G.; FACCINI, A. 2011. Caracterización microbiológica de Salmonella en alimentos de venta callejera en un sector universitario de Bogotá, Colombia. Julio a octubre de 2010. Médicas UIS. 24:26-33.

14. MERINO, L. 2008. Importancia de los vegetales que se consumen crudos en la transmisión de enfermedades de origen alimentario. Disponible desde Internet en: http: www.siicsalud.com-des-des043-05504016. htlm (con acceso 03/05/09).

15. NATARO J.; BOPP, C.; FIELDS, P.; KAPER, J.; STROCKBINE, N. 2007. Escherichia, Shigella y Salmonella. In: Murray PR, Baron E, Jorgensen J, Landry M., Pfaller MA. Manual of Clinical Microbiology. 9th ed. Washington, DC: ASM Press. p.670-687.

16. PEDREGAL, F.; GARCÍA, M.; NAVARRO, A.; LÓPEZ, F. 2002. Estudio microbiológico y condiciones sanitarias de los comedores no permanentes en Albacete, España. Centro de Salud. 10(6):330-332.

17. PERALTA, M.L.; AYALA, J. 2008. Algunas consideraciones sobre la prevalencia actual de Entamoeba histolytica, Giardia duodenalis, coccidios, microsporidios y mixosporidios en Colombia. Salud Uninorte. 24(2):294-302.

18. PÉREZ, G.; ROSALES, M.; VALDÉZ, R.; VARGAS, F.; CORDOVA, O. 2008. Detección de parásitos intestinales en agua y alimentos de Trujillo, Perú. Rev. Per. Med. Exp. Salud Pública. 25(1):144-148.

19. PORT, A.; ANTUNES, C.; MACHADO, O. 2004. Hospital food handlers in Niteroi, RJ, Brazil: Intestinal parasitism. Arch. Latinoam. Nutr. 54:395-401.

20. QUISPE, J.; SÁNCHEZ, V. 2001. Evaluación microbiológica y sanitaria de puestos de venta ambulatoria de alimentos del distrito de Comas, Lima, Perú. Rev. Per. Med. Exp. Salud Pública. 72:67-75. 
21. RINCÓN, D.; RAMÍREZ, R.; VARGAS, J. 2011. Transmisión de Salmonella enterica a través de huevos de gallina y su importancia en salud pública. Rev. Univ. Ind. Santander. Salud. 43(2):167-177.

22. RINCÓN, C.; GARZÓN, P.; GUASMAYAN, L.; FLÓREZ, A. 2009. Frecuencia de parasitismo intestinal en manipuladores de alimentos de cinco ciudades de Colombia, 2008. NOVA- Publ. Cient. Cienc. Bioméd. 7:80-84.

23. ROBERTS, D.; HOOPER, W.; GREENWOOD, M. 2000. Microbiología práctica de los alimentos. Ed. Acribia, S.A. (España). p.276.

24. RODRÍGUEZ, C.; PRADO, C. 2006. Microbiología: lo esencial y lo práctico. Organización Panamericana de la Salud (E.U). 248p.

25. RODULFO, H.; DE DONATO, M.; LUIGGI, J.; MICHELLI, E.; MILLÁN, A.; MICHELLI, M. 2012. Molecular characterization of Salmonella strains in individuals with acute diarrhea syndrome in the State of Sucre, Venezuela. Rev. Soc. Bras. Med. Trop. 45(3):329333.

26. SÁNCHEZ, C. 2006. Ensayo: Una mirada a las enfermedades parasitarias en el país. NOVA. 4:100-103.

27. URIBE, C.; SUÁREZ, M. 2006. Salmonelosis no tifoidea y su transmisión a través de alimentos de origen aviar. Col. Med. 37:151-158.
28. VALDIVIESO, N.; VILLALOBOS, L.; MARTÍNEZ, R. 2006. Evaluación microbiológica en manipuladores de alimentos de tres comedores públicos en Cumana, Venezuela. Rev. Soc. Ven. Microbiol. 26(2):95100.

29. VARGAS, J.; CLAVO, N.; MÁTTAR, S. 2004. Detección de Escherichia coli 0 157: H7 y Salmonella spp en cerdos del departamento de Córdoba. Rev. MVZ Córdoba. 9(1):386-392.

30. VÁSQUEZ, G.; GÓMEZ, E.; GAMBOA, E. 2007. Condiciones higiénico sanitarias de los servicios de alimentación en Instituciones infantiles del Instituto Colombiano de Bienestar Familiar de Bucaramanga, Colombia. Rev. Cub. Aliment. Nutr. 17:23-33.

31. VÁZQUEZ T., O; CAMPOS R., T. 2009. Giardiasis. La parasitosis más frecuente a nivel mundial. Rev. Centro Invest. U. La Salle (México). 8(31):75-90.

32. YÁNEZ, E.; MÁTTAR, S.; DURANGO, A. 2008. Determinación de Salmonella spp por PCR en tiempo real y método convencional en canales de bovinos y en alimentos de la vía pública de Montería, Córdoba. Infectio. 12(4):246-253.

Recibido: Julio 16 de 2012

Aceptado: Octubre 18 de 2012 